\title{
A METHOD OF ANALYTIC CONTINUATION SUGGESTED BY HEURISTIC PRINCIPLES
}

\author{
H. BRUYNES AND G. RAISBECK ${ }^{1}$
}

Suppose we are given an analytic function $f(z)$ represented by a power series (supposed convergent for some $z \neq 0$ )

$$
f(z)=\sum_{n=0}^{\infty} \frac{f_{n}}{n !} z^{n}
$$

where $f_{n}$ is the value of the $n$th derivative of $f(z)$ at the origin. For small values of $\delta$ we can approximate $f(\delta)$ in the following manner:

$$
f(\delta) \simeq f_{0}+\delta f_{1} .
$$

Refinement of this approximation leads to Taylor's theorem and back to the power series (1). It is possible, however, to use the linear approximation in a different way: we can use such approximations to go from one point to another along a chain of points $z=\delta, 2 \delta$, $3 \delta, \cdots, n \delta$. Thus we shall say

$$
f(\delta) \simeq f_{0}+\delta f_{1},\left.\quad f^{\prime}(z)\right|_{z=\delta} \simeq f_{1}+\delta f_{2},
$$

and so on, and

$$
f(2 \delta) \simeq f(\delta)+\left.\delta f^{\prime}(z)\right|_{z=\delta} \simeq f_{0}+2 \delta f_{1}+\delta^{2} f_{2} .
$$

In general

$$
f(n \delta) \simeq \sum_{m=0}^{n} a_{n, m} f_{m} \delta^{m} .
$$

It is easily verified that $a_{n, m}=a_{n-1, m}+a_{n-1, m-1}$ and hence that $a_{n, m}$ is the binomial coefficient $C_{n, m}$. If we now define the following:

$$
\sigma_{n}(z)=\sum_{m=0}^{n} f_{m} C_{n, m}\left(\frac{z}{n}\right)^{m}
$$

and if $n \delta=z$, then (3) is equivalent to

$$
f(z) \simeq \sigma_{n}(z) .
$$

The question now presents itself: for what values of $z$ does the sequence of polynomials $\sigma_{n}(z)$ converge to the function $f(z)$ ? It is evident that if $z$ is inside the circle of convergence of the series (1), then

Received by the editors February 17, 1948.

1 The authors are indebted to Prof. R. Salem for help and advice. 
we may say directly

$$
\begin{aligned}
\lim _{n \rightarrow \infty} \sigma_{n}(z) & =\lim _{n \rightarrow \infty} \sum_{m=0}^{n} f_{m} C_{n, m}\left(\frac{z}{n}\right)^{m} \\
& =\lim _{n \rightarrow \infty} \sum_{m=0}^{n} \frac{f_{m} z^{m}}{m !} \frac{n !}{(n-m) ! n^{m}} \\
& =\sum_{m=0}^{\infty} \frac{f_{m} z^{m}}{m !}=f(z) .
\end{aligned}
$$

However, this is not the complete answer.

It was shown by Borel $^{2}$ that we can without loss of generality restrict ourselves to the special case $f(z)=1 /(1-z)$. Here

$$
\begin{aligned}
f_{n} & =n ! \\
\sigma_{n}(z) & =\sum_{m=0}^{n} \frac{n !}{(n-m) !}\left(\frac{z}{n}\right)^{m} .
\end{aligned}
$$

Obviously if $|z|<1$

$$
\lim _{n \rightarrow \infty} \sigma_{n}(z)=\sum_{m=0}^{\infty} z^{m}=\frac{1}{1-z}
$$

and the convergence is uniform if $|z| \leqq 1-\epsilon$. It will be deduced later that the same is true if $|z| \leqq 1$ except for a neighborhood of the point $z=1$. Hence we shall confine ourselves to values of $z$ such that $|z|>1$. From (7)

$$
\begin{aligned}
\sigma_{n}(z) & =\sum_{m=0}^{n} \frac{n !}{(n-m) !}\left(\frac{z}{n}\right)^{m} \\
& =n !\left(\frac{z}{n}\right)^{n} \sum_{p=0}^{n} \frac{1}{p !}\left(\frac{n}{z}\right)^{p} \\
& =n !\left(\frac{z}{n}\right)^{n} e^{n / z}-\sum_{p=n+1}^{\infty} \frac{n !}{p !}\left(\frac{n}{z}\right)^{p-n}, \\
\sigma_{n}(z) & =n !\left(\frac{z}{n}\right)^{n} e^{n / z}-\sum_{r=1}^{\infty} z^{-r} \frac{n ! n^{r}}{(n+r) !} .
\end{aligned}
$$

If

$$
|z|>1
$$

2 Borel, Leçons sur les series divergentes, Paris, 1924, pp. $198 \mathrm{ff}$. 
then

$$
\lim _{n \rightarrow \infty}-\sum_{r=1}^{\infty} z^{-r} \frac{n ! n^{r}}{(n+r) !}=-\sum_{r=1}^{\infty} z^{-r}=\frac{1}{1-z}
$$

and the convergence is uniform if $|z| \geqq 1+\epsilon$. Using Stirling's formula, we have

$$
\lim _{n \rightarrow \infty} n !\left(\frac{z}{n}\right)^{n} e^{n / z}=\lim _{n \rightarrow \infty}(2 \pi n)^{1 / 2}\left(z e^{-1+1 / z}\right)^{n}
$$

This is zero if

$$
\left|z e^{-1+1 / z}\right|<1
$$

If we let $z=\rho e^{i \theta}$ then

$$
\left|z e^{-1+1 / z}\right|=\rho e^{-1+(\cos \theta) / \rho}
$$

and (13) is the same as

$$
\rho e^{-1+(\cos \theta) / \rho}<1
$$

or

$$
\cos \theta<\rho(1-\log \rho)
$$

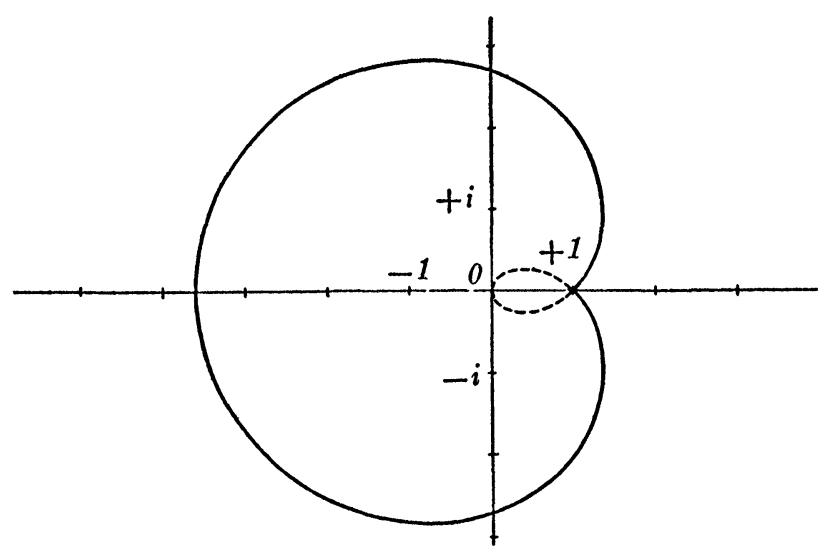

The curve $\cos \theta=\rho(1-\log \rho)$ and the region $S$. The region $S$ is bounded by the solid part of the curve.

We shall call the region bounded by the curve $\cos \theta=\rho(1-\log \rho)$, $\rho \geqq 1$, the region $S$ (see figure). In view of (8)-(12) and (14) we see that this is the region in which $\sigma_{n}(z) \rightarrow 1 /(1-z)$ uniformly, except 
possibly for a neighborhood of $|z|=1$ and a neighborhood of the boundary.

For the purpose of applying Borel's theorems it is also necessary to show that in the region $\left(1-\epsilon \leqq \rho \leqq 1+\epsilon, \epsilon^{\prime} \leqq \theta \leqq 2 \pi-\epsilon^{\prime}\right), \sigma_{n}(z)$ is bounded uniformly in $z$. This follows for $\rho \leqq 1$ from (7), by an application of Abel's transformation to the series $\sum_{0}^{\infty} z^{n}$ with the coeffcients (obviously monotonically decreasing) $n ! /(n-m) ! n^{m}$. Similarly it follows from (9) for $\rho \geqq 1$.

Borel $^{3}$ now proceeds as follows: suppose we have a system of polynomials $\sigma_{n}(z)=c_{0}^{(n)}+c_{1}^{(n)} z+c_{2}^{(n)} z^{2}+\cdots$ and such that $\sigma_{n}(z)$ $\rightarrow 1 /(1-z)$. Suppose we have also a function $f(z)$ represented by the power series

$$
\sum_{n=0}^{\infty} \frac{f_{n}}{n !} z^{n}
$$

We may write Cauchy's integral thus:

$$
f(z)=\frac{1}{2 \pi i} \int_{c} \frac{f(x)}{x} \frac{1}{1-z / x} d x
$$

where $c$ is a contour around $z$ not including any singularities of $f(z)$. Let

$$
F_{n}(z)=\frac{1}{2 \pi i} \int_{c} \frac{f(x)}{x} \sigma_{n}\left(\frac{z}{x}\right) d x .
$$

Borel showed that

$$
F_{n}(z)=\sum_{k=0}^{\infty} c_{k}^{(n)} \frac{f_{k}}{k !} z^{k}
$$

In case $\sigma_{n}(z) \rightarrow 1 /(1-z)$ uniformly in a certain star-shaped region including the circle of convergence of $\sum_{0}^{\infty} z^{n}$ Borel deduces by appropriate choice of the contour $c$ a region in which $F_{n}(z) \rightarrow f(z)$ which we describe as follows:

Construct for each singularity $\zeta$ of $f(z)$ a region $R(\zeta)$ similar to that in which $\sigma_{n}(z)$ converges to $1 /(1-z)$, such that the point corresponding to the origin is at the origin and the point corresponding to $z=1$ is at the singularity $\zeta$. Let $R=\prod_{5} R(\zeta)$ be the region common to all the $R(\zeta)$. In any finite region $R^{\prime}$ interior to $R$

$$
F_{n}(z) \rightarrow f(z)
$$

and the convergence is uniform if it is uniform in the special case

\footnotetext{
${ }^{3}$ Borel, loc. cit. We have changed the notation to agree with our own.
} 
$f(z)=1 /(1-z), F_{n}(z)=\sigma_{n}(z)$.

In our case, $c_{k}^{(n)}=n ! /(n-k) ! n^{k}, \sigma_{n}(z) \rightarrow 1 /(1-z)$ uniformly in any subregion of $S$ interior to $S$ except in a region as small as we please where $\left|\sigma_{n}(z)-1 /(1-z)\right|$ is uniformly bounded. The reader may convince himself that this restriction does not alter Borel's results.

If in the identity (9) we replace $z$ by $1 / z$ we can derive a more powerful method of continuation. It is not, however, as powerful as those of Borel, Mittag-Leffler, and others, and hence we shall not discuss it.

United Aircraft Corporation,

New College, Oxford University, and

MassachusetTs Institute of TEChNOLOGY 